\title{
National Outcomes of the Fontan Operation with Endocardial Cushion Defect
}

\author{
Sandeep Sainathan ${ }^{1}$, Sameh Said ${ }^{2}$, Chris Agala ${ }^{3}$, Leonardo Mulinari ${ }^{1}$, and Mahesh \\ Sharma ${ }^{3}$ \\ ${ }^{1}$ University of Miami Department of Surgery \\ ${ }^{2}$ University of Minnesota Division of Cardiothoracic Surgery \\ ${ }^{3}$ University of North Carolina at Chapel Hill Libraries
}

January 27, 2022

\begin{abstract}
Objective: The traditional outcomes of the Fontan Operation (FO) in Endocardial Cushion Defect (ECD) patients have been suboptimal. Previous studies have been limited by the smaller number of ECD patients, longer study period with an era effect and do not directly compare short-term outcomes of FO in ECD patients with non-ECD patients. Our study aims to address these shortcomings. Methods: A retrospective analysis of the Kids Inpatient Database (2009, 2012, and 2016) for the FO was done. The groups were divided into those who underwent FO with ECD as compared to non-ECD diagnosis. The data was abstracted for demographics, clinical characteristics, and operative outcomes. Standard statistical tests were used. Results: 3380 patients underwent the FO of which 360 patients (11\%) were FO-ECD. ECD patients were more likely to have Down syndrome, Heterotaxy syndrome, transposition/DORV, and TAPVR as compared to non-ECD patients. FO-ECD had a higher discharge-mortality $(2.84 \%$ vs. $0.45 \%, \mathrm{p}=0.04)$. The length of stay ( 16 vs. 13 days, $\mathrm{p}=0.05)$ and total charges incurred $(\$ 283,280$ vs. $234,106, \mathrm{p}=0.03)$ for the admission were higher in the FO-ECD as compared to non-ECD patients. In multivariable analysis: ECD diagnosis, cardiac arrest, acute kidney injury, and post-operative hemorrhage were predictors of mortality. Conclusion: Contemporary outcomes for FO are excellent with very low overall operative mortality. However, the outcomes in ECD patients are inferior with higher operative mortality than non-ECD patients. Occurrence of post-operation complications, associated TAPVR and a diagnosis of ECD were predictive of a negative outcome.
\end{abstract}

\section{Hosted file}

Manuscript Fontan ECD Final.docx available at https://authorea.com/users/448755/articles/ 554333-national-outcomes-of-the-fontan-operation-with-endocardial-cushion-defect 